\title{
IGF2BP2 Overexpression Indicates Poor Survival in Patients with Acute Myelocytic Leukemia
}

\author{
Xin $\mathrm{He}^{\mathrm{a}}$ Wenlu Li ${ }^{\mathrm{b}} \quad$ Xiaogong Liang ${ }^{\mathrm{c}} \quad$ Xuan Zhu $^{\mathrm{d}} \quad$ Lei Zhang ${ }^{\mathrm{a}}$ \\ Yu Huange Teng Yu ${ }^{\mathrm{a}}$ Shu Li ${ }^{\mathrm{a}}$ Zhigang Chen ${ }^{\mathrm{d}, \mathrm{f}}$ \\ aDepartment of Hematology, the Second Affiliated Hospital, Zhejiang University School of Medicine, \\ Hangzhou, 'Department of Pharmacy, the Second Affiliated Hospital, Zhejiang University School of \\ Medicine, Hangzhou, 'Department of Hematology, Central Hospital of MianYang city, Mianyang, \\ dDepartment of Surgical Oncology, the Second Affiliated Hospital, Zhejiang University School of \\ Medicine, Hangzhou, eDepartment of Experimental Animal Research Center, Zhejiang Chinese Medical \\ University, Hangzhou, fKey Laboratory of Tumor Microenvironment and Immune Therapy of Zhejiang \\ Province, Hangzhou, China
}

\section{Key Words}

IGF2BP2 • Acute myelocytic leukemia • Prognosis • Proliferation

\begin{abstract}
Background/Aims: IGF2BP2 has been reported to serve as an oncogene in various solid cancers. However, the role of IGF2BP2 in acute myelocytic leukemia (AML) is still unknown. Methods: Public databases Gene Omnibus was used to evaluate the expression of IGF2BP2 in AML patients and healthy controls. In addition, primary cells from these two populations were prepared by Ficoll density centrifugation. Rt-qPCR and western blot were used to detect IGF2BP2 expression in the primary cells from these two populations. Meta-analysis was performed to evaluate the association of IGF2BP2 and prognosis. Lentivirus-based shRNAs were used to knock down IGF2BP2 in AML cell lines KG-1a and Kasumi. Results: We searched the public database Gene Omnibus and analyzed IGF2BP2 expression in both AML and healthy populations. The results showed that IGF2BP2 was overexpressed in AML patients. To verify this phenomenon in fresh human samples, we compared the expression of IGF2BP2 in primary cells from $10 \mathrm{AML}$ patients and 10 healthy controls and found that the expression of IGF2BP2 was upregulated in AML primary cells. More importantly, we observed that IGF2BP2 expression was negatively correlated with the CEBPA mutation status, which is an indicator of good prognosis ( $R R=0.648, p=0.0001)$. In addition, IGF2BP2 expression was positively associated with poor prognostic factors, such as the FLT3-ITD mutation $(R R=1.198, p=0.0009)$ and IDH1 mutation $(\mathrm{RR}=1.354, \mathrm{p}=0.0003)$, as well as intermediate and poor cytogenetic risk $(R R=1.214, p=0.0026)$. To evaluate the prognostic value of IGF2BP2 in $A M L$, we further performed a meta-analysis of 8 studies consisting of 1731 patients and found that IGF2BP2

X. He, W. Li and X. Liang contributed equally to this work.

Zhigang Chen

Department of Surgical Oncology, the Second Affiliated Hospital, Zhejiang University School of Medicine, Hangzhou, 310009 (China)

E-Mail chenzhigang@zju.edu.cn
\end{abstract}

KARGER 
overexpression was correlated with worse overall survival in $A M L$ patients [HR=1.31(1.161.49); $p=0.00]$. Furthermore, we performed Gene Omnibus and Gene Set Enrichment analyses and found that the genes regulated by IGF2BP2 were mainly enriched in cell proliferation. IGF2BP2 knockdown by 4 different shRNA vectors significantly inhibited the growth of two AML cell lines, KG-1a and Kasumi. Conclusion: Thus, IGF2BP2 may serve as a biomarker to predict the prognosis of $A M L$ and as a potential target in AML.

\section{Introduction}

Acute myelocytic leukemia (AML) is a common malignant disease of the hematopoietic system [1, 2]. According to statistics reported by the American Cancer Society in 2016, approximately 19, 950 new cases and 10, 430 deaths from AML are expected in the United States [3]. AML is characterized by high heterogeneity, and its prognosis varies greatly. Mutations in CEBPA, NPM1, FLT3, MLL, IDH1 or IDH2, DNMT3A, and ASXL1 have been suggested to predict the outcome of patients with AML $[4,5]$. However, these biomarkers are based on the gene mutations of patients. It is necessary to identify new biomarkers to predict the prognosis of AML and develop personalized treatments.

IGF2 mRNA binding protein 2 (IGF2BP2) is an RNA-binding protein that plays an important role in regulating the differentiation potentials of mouse neocortical neural precursor cells as well as myoblast proliferation, myogenesis, muscle cell motility and energy metabolism [6-8]. IGF2BP2 has been recently reported to be overexpressed in several types of solid cancers $[9,10]$. IGF2BP2 overexpression promotes glioblastoma multiforme (GBM) progression by activating the IGF2/PI3K/Akt pathway and makes GBM resistant to temozolomide treatment [11]. In addition, IGF2BP2 promotes hepatocarcinogenesis by inducing inflammation [12]. Moreover, elevated IGF2BP2 is associated with poor survival in esophageal adenocarcinoma and basal-like breast cancer patients $[9,10]$. Therefore, IGF2BP2 is a potential oncogene and prognostic marker in solid cancers. However, the role of IGF2BP2 in AML has not yet been investigated.

In this study, we first investigated the role of IGF2BP2 expression in AML by in silico analysis and tested its prognostic significance. By searching public datasets, we found that IGF2BP2 is upregulated in AML patients and significantly associated with poor overall survival in AML patients. In addition, IGF2BP2 knockdown significantly inhibits the growth of AML cell lines. Thus, IGF2BP2 could serve as a potential prognostic marker and promising therapeutic target in AML patients.

\section{Materials and Methods}

Primary cells from healthy controls and AML patients

Ten healthy controls and ten AML patients were included in this study, and informed consent was obtained from all participants. Primary mononuclear cells were collected from the bone marrow samples of healthy controls and AML patients by Ficoll density centrifugation. To better represent the characteristics of AML, the leukemia blasts from AML patients had an $85 \%$ or higher purity.

Quantitative Real-time Polymerase Chain Reaction (qRT-PCR)

RNA was isolated from primary patients with AML and healthy controls according to the protocol of an RNeasy Mini Kit (Qiagen \#74104) and was then used to synthesize cDNA by using a PrimeScript ${ }^{\text {TM }}$ RT Reagent Kit with a gDNA Eraser (TaKaRa \#RR047A). qRT-PCR was performed with a SYBR® Green Real-time PCR Master Mix kit (TOYOBO \#QRT-201). The reaction volume of the qRT-PCR reactions was $20 \mu l$, containing

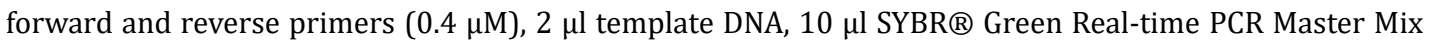
and $6.4 \mu \mathrm{l}$ PCR-grade water. qRT-PCR was performed on a Roche LightCycler ${ }^{\mathrm{TM}}$, and the cycling conditions were as follows: $95^{\circ} \mathrm{C}$ for $30 \mathrm{sec}$, followed by 40 cycles at $95^{\circ} \mathrm{C}$ for $5 \mathrm{sec}, 60^{\circ} \mathrm{C}$ for $10 \mathrm{sec}$ and $72^{\circ} \mathrm{C}$ for 15 sec. GAPDH was used as the housekeeping gene. Amplification efficiencies for IGF2BP2 and GAPDH primer 


\section{Cellular Physiology Cell Physiol Biochem 2018;51:1945-1956 and Biochemistry Published \begin{tabular}{l|l} 
DOI: 10.1159/000495719 & $\begin{array}{l}\text { @ } 2018 \text { The Author(s). Published by S. Karger AG, Basel } \\
\text { www.karger.com/cpb }\end{array}$
\end{tabular}}

He et al.: The Role of IGF2BP2 in Acute Myelocytic Leukemia

sets were detected using ten-fold serial dilutions of KG-1a cell line cDNA. All the primers were designed online by PrimerBank (https://pga.mgh.harvard.edu/primerbank/). IGF2BP2 forward primer sequence: AGTGGAATTGCATGGGAAAATCA; reverse primer sequence: CAACGGCGGTTTCTGTGTC. GAPDH forward primer sequence: GGAGCGAGATCCCTCCAAAAT; reverse primer sequence: GGCTGTTGTCATACTTCTCATGG.

\section{Western blotting}

In brief, cellular proteins were extracted from the primary cells or cell lines and separated by SDSPAGE. The proteins in the gel were then transferred onto polyvinylidene fluoride (PVDF) membranes (Bio-Rad, Hercules, CA). The membranes were blocked in 5\% BSA/TBST for 1 hour and then washed with TBST and incubated with a primary rabbit anti-IGF2BP2 antibody (Abcam ab124930) and primary rabbit anti-GAPDH antibody (Abcam ab181602) at $4^{\circ} \mathrm{C}$ overnight. The membranes were then washed with TBST and incubated with a secondary goat anti-rabbit IgG antibody (Abcam ab6721). Finally, the membranes were washed with TBST, and the protein expression levels were detected by chemiluminescence (Thermo ScientificTM \#32104).

\section{Knockdown of IGF2BP2}

Lentivirus-based shRNAs targeting IGF2BP2 (NM_001007225) were purchased from GeneChem (28870-28873, Shanghai, China). The targeting coding sequences of IGF2BP2 were as follows: IGF2BP2 shRNA1, 5'-TAACCAGTGCAGAAGTCAT-3'; IGF2BP2 shRNA2, 5'- GATCTTTGGGAAACTGAAA-3'; IGF2BP2 shRNA3, 5'-GTGCTGAGATAGAGATTAT-3'; IGF2BP2 shRNA4, 5'- ATATACAACCCGGAAAGAA-3'. Lentivirus transduction was performed according to previously described methods [14]. Briefly, $2 \mathrm{ml}$ of virus was added to the cells containing $5 \mu \mathrm{g} / \mathrm{ml}$ protamine sulfate, followed by overnight coculture. The cells were centrifuged and washed with PBS and then cultured in fresh medium with $2 \mu \mathrm{g} / \mathrm{ml}$ puromycin for three days. The cells were then collected to detect the mRNA and protein expression of IGF2BP2.

\section{Cell proliferation assay}

The AML cell lines KG-1a and Kasumi were purchased from the American Type Culture Collection (ATCC) and cultured in RPMI 1640 medium supplemented with 10\% fetal bovine serum (Gibco, Grand Island, NY). A total of $2 \times 10^{3} \mathrm{KG}-1 \mathrm{a}$ and Kasumi cells were seeded in $96-$ well plates and cultured at $37^{\circ} \mathrm{C}$. The cells were divided into five groups, including one shControl group and four shRNA groups. Each group had five replicates. Cell proliferation was determined by CCK-8 assays (Dojindo Lot.JE603) at various time points according to the methods of the manufacturer.

\section{Gene Omnibus (GEO) dataset analysis}

GEO datasets (GSE13159, GSE7186, GSE48558) were downloaded, and the normalized data were used to analyze the differential expression of IGF2BP2 in AML patients and healthy controls. The GEO dataset (GSE6891) containing 521 AML cases was chosen to test the relationship between IGF2BP2 expression and the clinicopathological features. Normalized and processed data from the RNA-seq sequencing GEO dataset (GSE80946) were analyzed to acquire the differentially expressed genes between the shRNA-targeting IGF2BP2 group and the shRNA control group. For the sets of differentially expressed genes, the enriched Gene Ontology terms were identified by using the DAVID program [15, 16]. Gene set enrichment analysis (GSEA) was also performed by using GSEA v2.0 software, which was available from the Broad Institute (http://www.broad.mit.edu/gsea) [17].

\section{Survival analysis and meta-analysis}

The survival analysis was based on the expression data of IGF2BP2 from various GEO datasets (GSE12417, GSE8043, GSE10358, GSE1427, GSE6891), and the clinical survival information was obtained from PREdiction of Clinical Outcomes from Genomic profiles (PRECOG) or provided by the author [19-25, 32]. After collecting the survival information and IGF2BP2 expression values, hazard ratio (HR) values were recalculated or obtained directly from PRECOG to perform the survival analysis. The median expression value was selected to define the cut-off value for high IGF2BP2 expression.

A meta-analysis was conducted to evaluate the overall association between IGF2BP2 expression and survival in patients with AML. The pooled HRs and their 95\% CIs were used in this meta-analysis. An observed HR value $>1$ indicated that high IGF2BP2 expression was correlated with poor survival in AML 
patients. If the $95 \% \mathrm{CI}$ value did not overlap with a value of 1 , the results were considered statistically significant. A heterogeneity analysis was carried out based on the Chi-square-based Q statistical test, and a $P$ value $>0.1$ implied no heterogeneity across the studies [33]. In such cases, a fixed-effect model (the Mantel-Haenszel method) was used to perform the meta-analysis instead of a random-effect model (the DerSimonian and Laird method)[33, 34]. Both Egger's test and Begg's test were adopted to calculate the publication bias, and a p value $<0.05$ indicated that bias existed.

\section{Statistical analysis}

Differential gene expression and a heat map analysis were carried out by using R software and GeneSpring GX. The statistical analyses of the meta-analysis were performed using STATA version 12.0 software (Stata Corporation, Collage Station, Texas, USA). An independent-sample t test was used to analyze the differential IGF2BP2 expression between the two groups, and one-way analysis of variance was used for comparing several groups. Log-rank tests were used to assess the prognostic value of IGF2BP2 expression. All the tests were two-sided and were considered statistically significant when the $p$ values were less than 0.05 .

\section{Results}

IGF2BP2 is overexpressed in human AML

To investigate the differences in IGF2BP2 expression between patients with AML and healthy controls, we analyzed the mRNA levels of IGF2BP2 in two separate patient cohort datasets (GSE7186 and GSE48558); IGF2BP2 expression was higher in AML patients than in healthy controls, especially in the GSE48558 dataset $(0.87 \pm 0.05$ vs $3.1 \pm 0.3$; a nearly 8 -fold

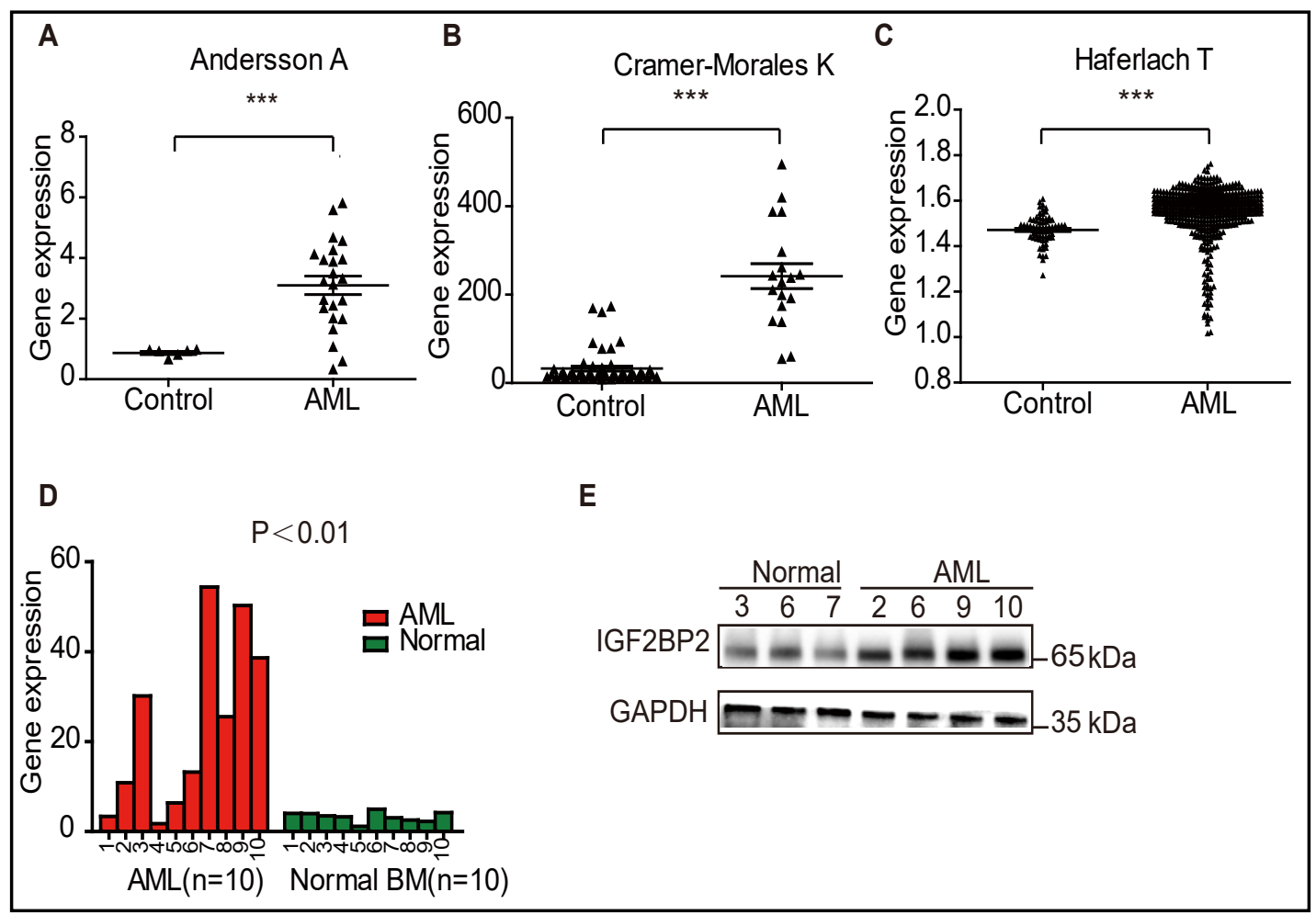

Fig. 1. IGF2BP2 is overexpressed in AML patients. (A-C) Gene expression analysis of IGF2BP2 in the AML GEO datasets GSE7186 (control=6, AML=23), GSE48558 (control=49, AML=18) and GSE13159 (control=73, AML=542); (D) qRT-PCR analysis of IGF2BP2 expression in primary AML patients ( $\mathrm{n}=10$ ) and healthy controls $(n=10)$; the results shown represent the means of triplicates; (E) Immunoblot of IGF2BP2 expression in healthy controls $(n=3)$ and primary AML patients $(n=4) .{ }^{* * *} \mathrm{p}<0.001$. 
Table 1. The clinical-pathological features of ten primary AML patients

\begin{tabular}{|c|c|c|c|c|c|c|}
\hline Patients & Subtype & Sex & WBC $(109 / \mathrm{L})$ & $\begin{array}{c}\text { Primary cells } \\
\text { Percentage (\%) }\end{array}$ & Gene mutation & Chromosome karyotype \\
\hline AML1 & M2 & Male & 66.8 & 94 & AML/ETO & $46, \mathrm{XY}, \operatorname{der}(3) \mathrm{t}(3 ; 8)(\mathrm{p} 26 ; \mathrm{q} 13), \mathrm{t}(8 ; 21)$ \\
\hline AML2 & M2 & Female & 39.5 & 87 & No & $47, \mathrm{XX},+\operatorname{mar}[16] / 46, \mathrm{XX}[4]$ \\
\hline AML3 & M5 & Female & 7 & 90 & No & $46, \mathrm{XX}, \mathrm{t}(8 ; 19)(\mathrm{q} 13 ; \mathrm{p} 13.1), \operatorname{del}(9)(\mathrm{q} 2 \mathrm{2q} 34 ; \operatorname{del}(11)(\mathrm{q} 23)[5]$ \\
\hline AML4 & M1 & Female & 6 & 95 & CEBPA & $47, \mathrm{X}, \mathrm{i}(\mathrm{X})(\mathrm{q} 10),+10[5] / 46, \mathrm{X},-\mathrm{X},+10[4]$ \\
\hline AML5 & M4 & Male & 2.5 & 88 & FLT3-ITD & $46, \mathrm{X},-\mathrm{Y},+8, \mathrm{t}(8 ; 21)(\mathrm{q} 22 ; \mathrm{q} 22)[18] / 46, \mathrm{XY}[2]$ \\
\hline AML6 & M5 & Male & 244.67 & 93 & FLT3-ITD,dupMLL & $46, \mathrm{XY}[8]$ \\
\hline AML7 & M4 & Male & 35.5 & 92 & CEBPA/MYH11 & $46, \mathrm{XY}, \operatorname{inv}(16)(\mathrm{p} 13,1 \mathrm{q} 22)[20]$ \\
\hline AML8 & CML-BC/M & Female & 23.9 & 90 & BCR/ABL(P210),AML1/MDS1,EVI1 & $46, \mathrm{XX}, \mathrm{t}(9,22)(\mathrm{q} 34 ; \mathrm{q} 11.2)[18] / 46$, idem, $\mathrm{t}(3 ; 21)(\mathrm{q} 26 ; \mathrm{q} 22)[2]$ \\
\hline AML9 & M5 & Male & 58 & 95 & EVI1 & $46, \mathrm{XY}, \mathrm{t}(11,19)(\mathrm{q} 23, \mathrm{p} 13.1)$ \\
\hline AML10 & M4 & Male & 17 & 86 & No & $46, \mathrm{XY}$ \\
\hline
\end{tabular}
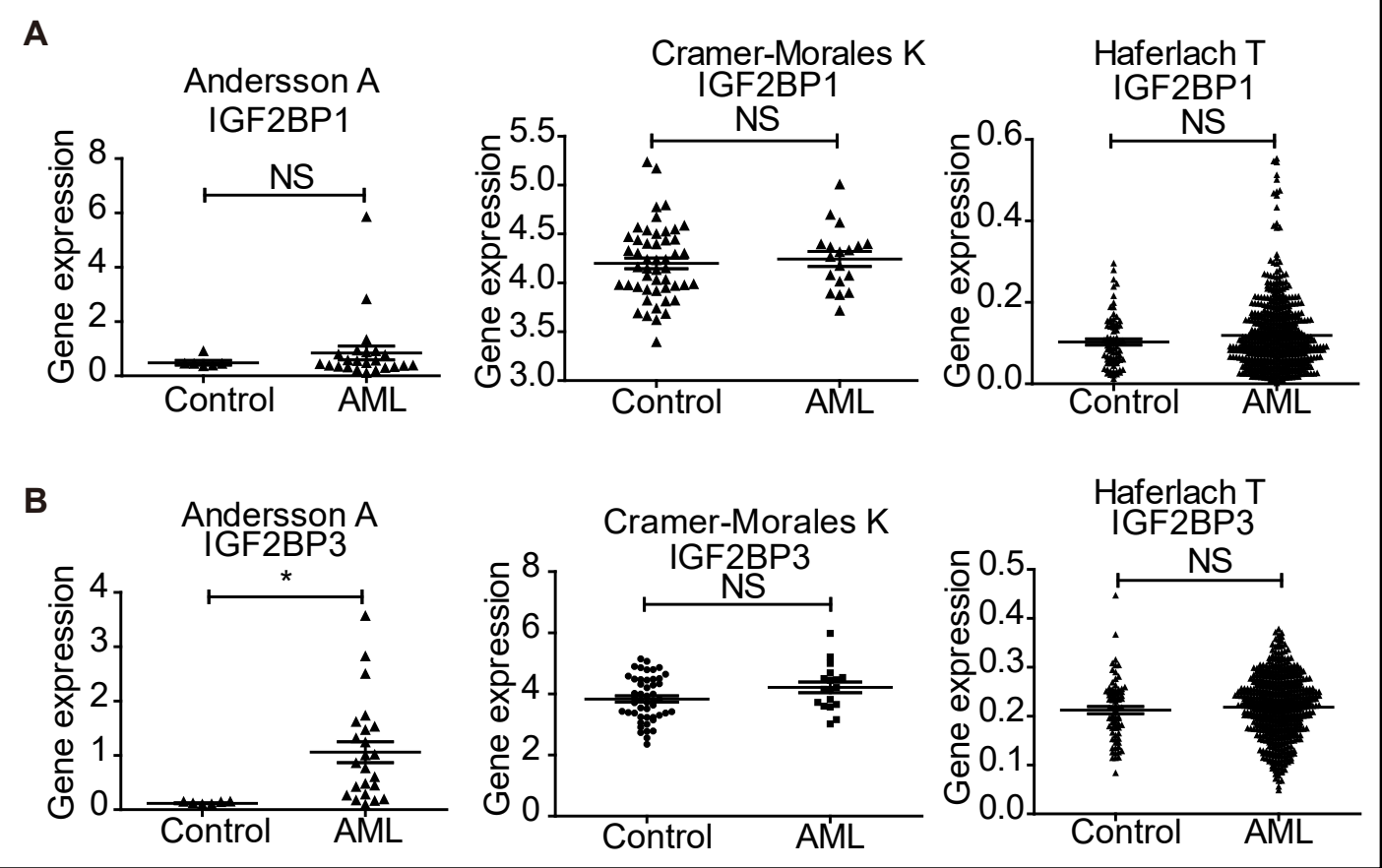

Fig. 2. The expression of IGF2BP1 and IGF2BP3 in AML patients and healthy controls. (A) Gene expression analysis of IGF2BP1 in three independent datasets (GSE7186 Anderson A, GSE48558 Cramer-Morales K and GSE13159 Haferlach T); (B) Gene expression analysis of IGF2BP3 in three independent datasets (GSE7186 Anderson A, GSE48558 Cramer-Morales K and GSE13159 Haferlach T). NS: not significant, * ${ }^{*}<0.05$.

increase)(Fig. 1A-B). Further analysis of the GSE13159 dataset, which contains 542 AML patients and 73 healthy controls, also showed that IGF2BP2 was overexpressed in AML patients (Fig. 1C). To verify this result in fresh human samples, we prepared primary cells from a cohort of ten primary AML patients and ten healthy volunteers. The clinical characteristics of these patients are shown in Table 1 . There were six male and four female patients, and the histopathologic subtypes of the patients included one M1, two M2, three M4, three M5 and one CML-BC/M. The percentage of primary cells isolated from AML patients ranged from $86 \%$ to $95 \%$. We then performed qRT-PCR and western blotting to determine the expression of IGF2BP2 both in AML patients and healthy controls. Since the number of cells isolated from AML patients varied greatly, we did not have sufficient material to perform a western blot analysis for all samples. Therefore, we simply chose the samples with enough cells to perform the Western blots. Furthermore, we performed RT-qPCR analysis instead of a western blot on the samples that did not have enough cells. The results showed that IGF2BP2 was significantly upregulated both at the mRNA and protein levels in the AML patients (Fig. 1D-E). Next, we asked whether the increased IGF2BP2 expression was associated with its gene amplification. According to the TCGA datasets (http://www.cbioportal.org/), we found that only 1 in 187 AML patients had IGF2BP2 gene amplification, and 3 had IGF2BP2 gene deletion. In addition, we also analyzed the mutation status of IGF2BP2 in these AML patients, and no IGF2BP2 
mutation was found. Therefore, the reason for the increased IGF2BP2 expression in AML may not be gene amplification or mutation. Furthermore, we have analyzed the expression of IGF2BP1 and IGF2BP3 in AML patients and healthy controls. The results showed that the expression of IGF2BP1 did not differ significantly in these two populations in the three independent datasets (GSE7186, GSE48558 and GSE13159) (Fig. 2A). The expression of IGF2BP3 in the AML patients and healthy controls was still controversial. The expression of IGF2BP3 was
Table 2. The association between IGF2BP2 expression and clinical-pathological features of AML

\begin{tabular}{lcc}
\hline Variable & IGF2BP2 expression value(mean) & P value \\
\hline Female & $425(\mathrm{n}=228)$ & 0.5515 \\
Male & $438(\mathrm{n}=230)$ & \\
Age $(\geq 50)$ & $432(\mathrm{n}=161)$ & 0.9965 \\
Age $(<50)$ & $432(\mathrm{n}=297)$ & \\
Cytogenetic good & $369(\mathrm{n}=97)$ & 0.0026 \\
Cytogenetic intermediate and poor & $448(\mathrm{n}=351)$ & \\
IDH2 negative & $431(\mathrm{n}=417)$ & 0.7307 \\
IDH2 positive & $448(\mathrm{n}=38)$ & \\
IDH1 negative & $421(\mathrm{n}=421)$ & 0.0003 \\
IDH1 positive & $569(\mathrm{n}=34)$ & \\
CEBPA wild type & $443(\mathrm{n}=424)$ & \\
CEBPA mutant & $287(\mathrm{n}=34)$ & \\
FLT3-ITD negative & $409(\mathrm{n}=333)$ & 0.0000 \\
FLT3-ITD positive & $489(\mathrm{n}=125)$ & \\
FLT3-TKD negative & $434(\mathrm{n}=408)$ & 0.3715 \\
FLT3-TKD positive & $404(\mathrm{n}=50)$ & \\
N-ras negative & $436(\mathrm{n}=412)$ & 0.1767 \\
N-ras positive & $387(\mathrm{n}=45)$ & \\
K-ras negative & $430(\mathrm{n}=454)$ & 0.3476 \\
K-ras positive & $539(\mathrm{n}=4)$ & \\
Evil negative & $435(\mathrm{n}=427)$ & 0.2091 \\
Evil positive & $381(\mathrm{n}=31)$ & \\
\hline
\end{tabular}

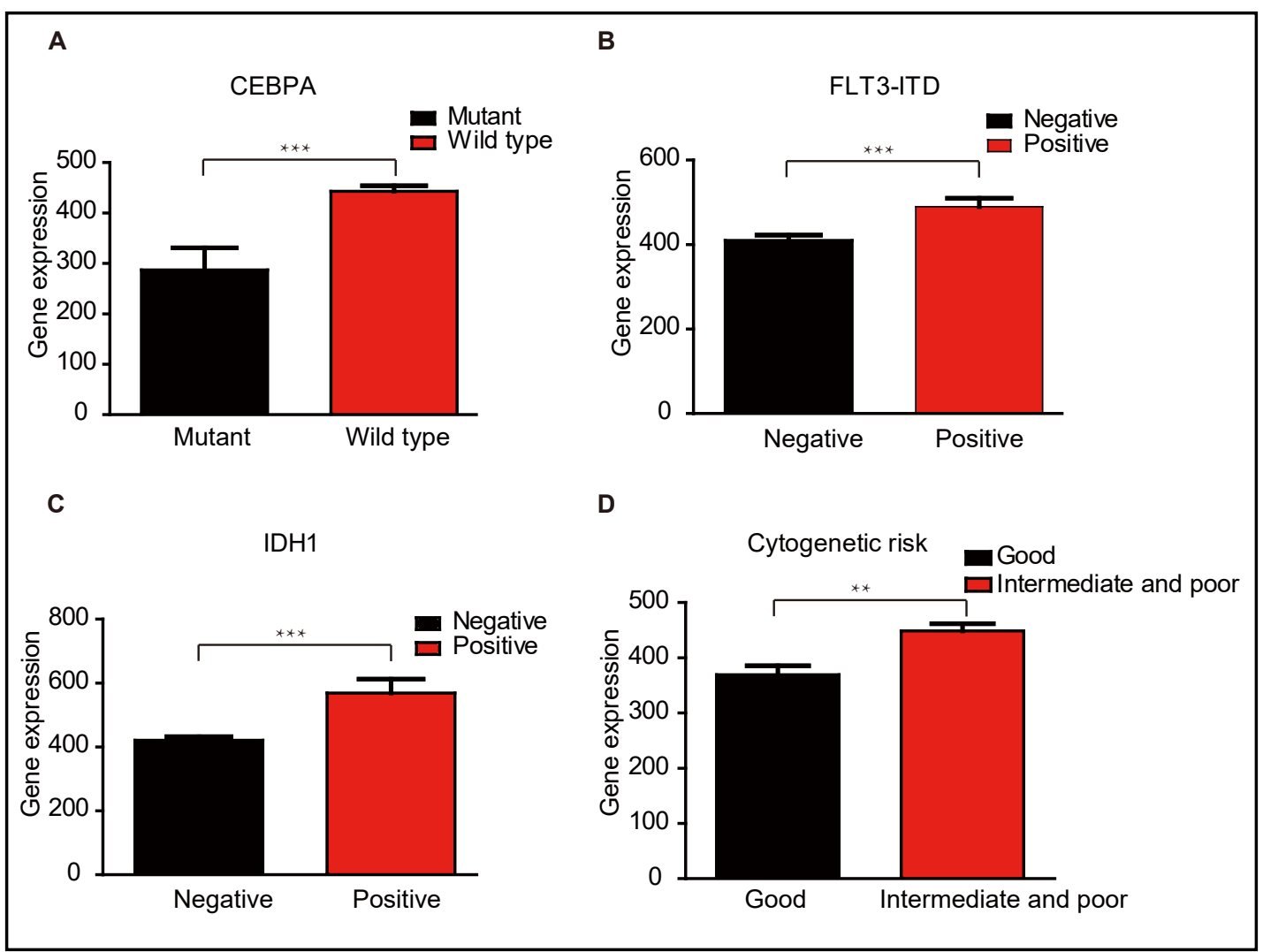

Fig. 3. High IGF2BP2 expression is associated with poor clinical-pathological features in AML patients. (A) Bar chart shows the mean \pm SEM gene expression of IGF2BP2 in AML patients with the CEBPA mutation status; (B) Bar chart shows the mean \pm SEM gene expression of IGF2BP2 in AML patients with FLT3-ITD mutation; (C) Bar chart shows the mean \pm SEM gene expression of IGF2BP2 in AML patients with IDH1 mutation; (D) Bar chart shows the mean \pm SEM gene expression of IGF2BP2 in AML patients with cytogenetic risk. ${ }^{*} \mathrm{p}<0.05,{ }^{* *} \mathrm{p}<0.01$, and ${ }^{* * *} \mathrm{p}<0.001$. 
higher in the AML patients than in the healthy controls (GSE7186), while there was no difference in the other two datasets (GSE48558 and GSE13159) (Fig. 2B). Taken together, IGF2BP2 rather than IGF2BP1 and IGF2BP3 is overexpressed in human AML.

Association of IGF2BP2 expression with the clinical characteristics of AML patients

To determine the relationship between IGF2BP2 expression and the clinical characteristics of AML patients, we investigated the relevant clinical data in a published dataset (GSE6891) containing 521 AML patients [13, 14]. As shown in Table 2, IGF2BP2 expression was not associated with sex, age or mutations, such as IDH2, FLT3-TKD, N-ras, K-ras and Evil ( $p>0.05)$. However, IGF2BP2 expression was negatively correlated with the CEBPA mutation status (mean value 287 for mutant vs 443 for wild type, $R R=0.648$, $\mathrm{p}=0.0001$ ), which is an indicator of good prognosis (Fig. 3A). In addition, IGF2BP2 expression was positively associated with poor prognostic factors, such as the FLT3-ITD mutation (mean value 490 for mutant vs 409 for wild type, $R R=1.198, p=0.0009$ ) (Fig. 3B) and IDH1 mutation (mean value 570 for mutant vs 421 for wild type, $R R=1.354, p=0.0003$ ) (Fig. 3C), as well as intermediate and poor cytogenetic risk (mean value 448 for intermediate and poor risk vs 369 for good risk, $R R=1.214, p=0.0026$ ) (Fig. 3D). These results indicated that IGF2BP2 expression may be associated with worse clinical characteristics in AML patients.

\section{IGF2BP2 expression correlates with the overall survival of AML patients}

Given the relationship between IGF2BP2 expression and prognostic factors, including the CEBPA, FLT3-ITD, and IDH1 mutation statuses and intermediate and poor cytogenetic risk, we next examined the prognostic significance of IGF2BP2 in patients with AML. We compared the overall survival (OS) of 173 AML patients with differential IGF2BP2 expression from the GSE10358 dataset [15]. The results showed that the IGF2BP2 overexpression group

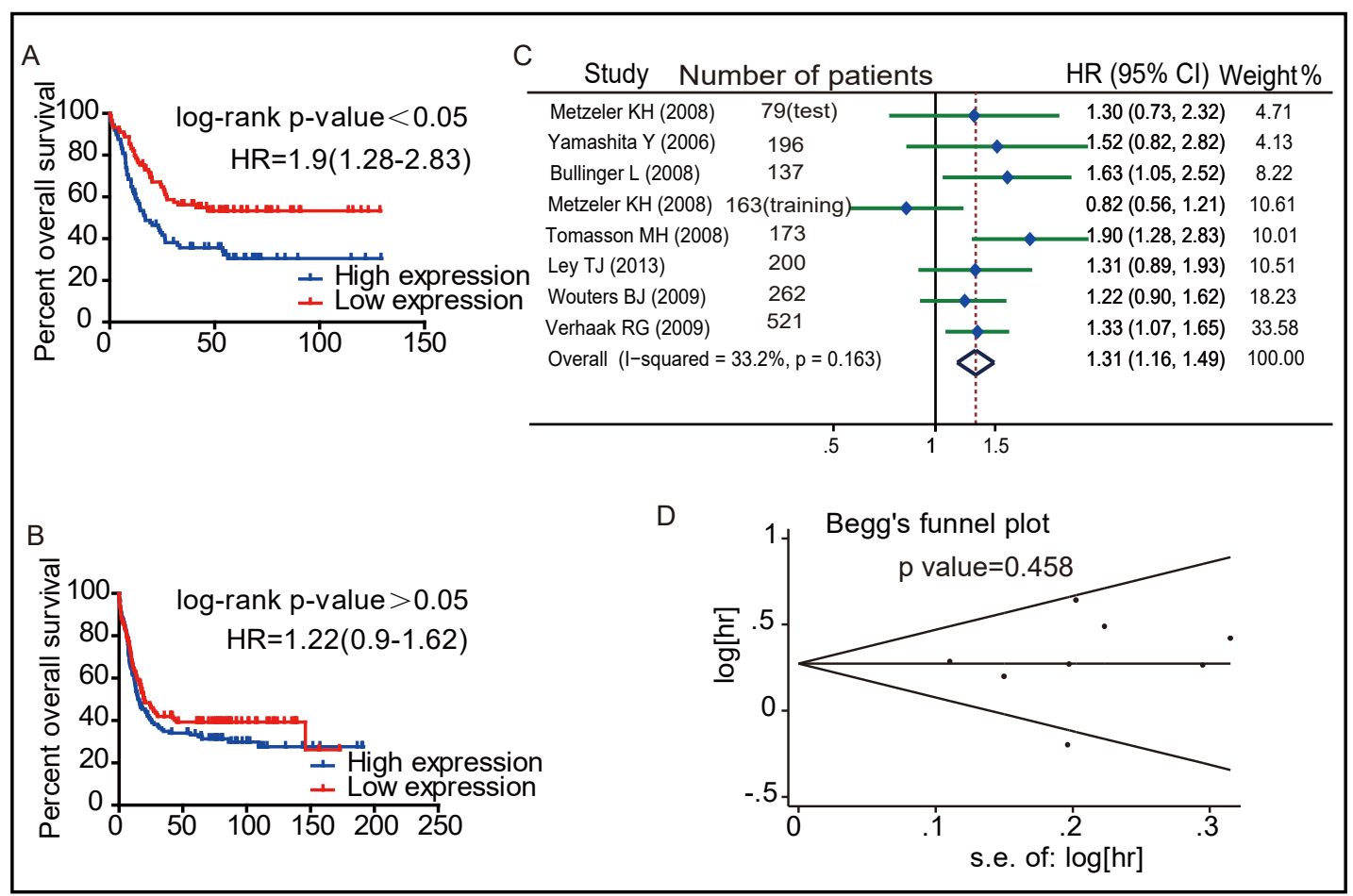

Fig. 4. High IGF2BP2 expression correlates with poor survival in AML patients. (A) Overall survival (OS) analysis of AML patients ( $n=173$ ) using the expression of IGF2BP2 (GSE10358); (B) OS analysis of AML patients ( $n=262$ ) using the expression of IGF2BP2 (GSE14468); (C) Forrest plot of hazard ratios (HRs) for the association of IGF2BP2 overexpression with OS; (D) Begg's publication bias plot. No publication bias was observed in the meta-analysis. Log-rank test (A-B). 


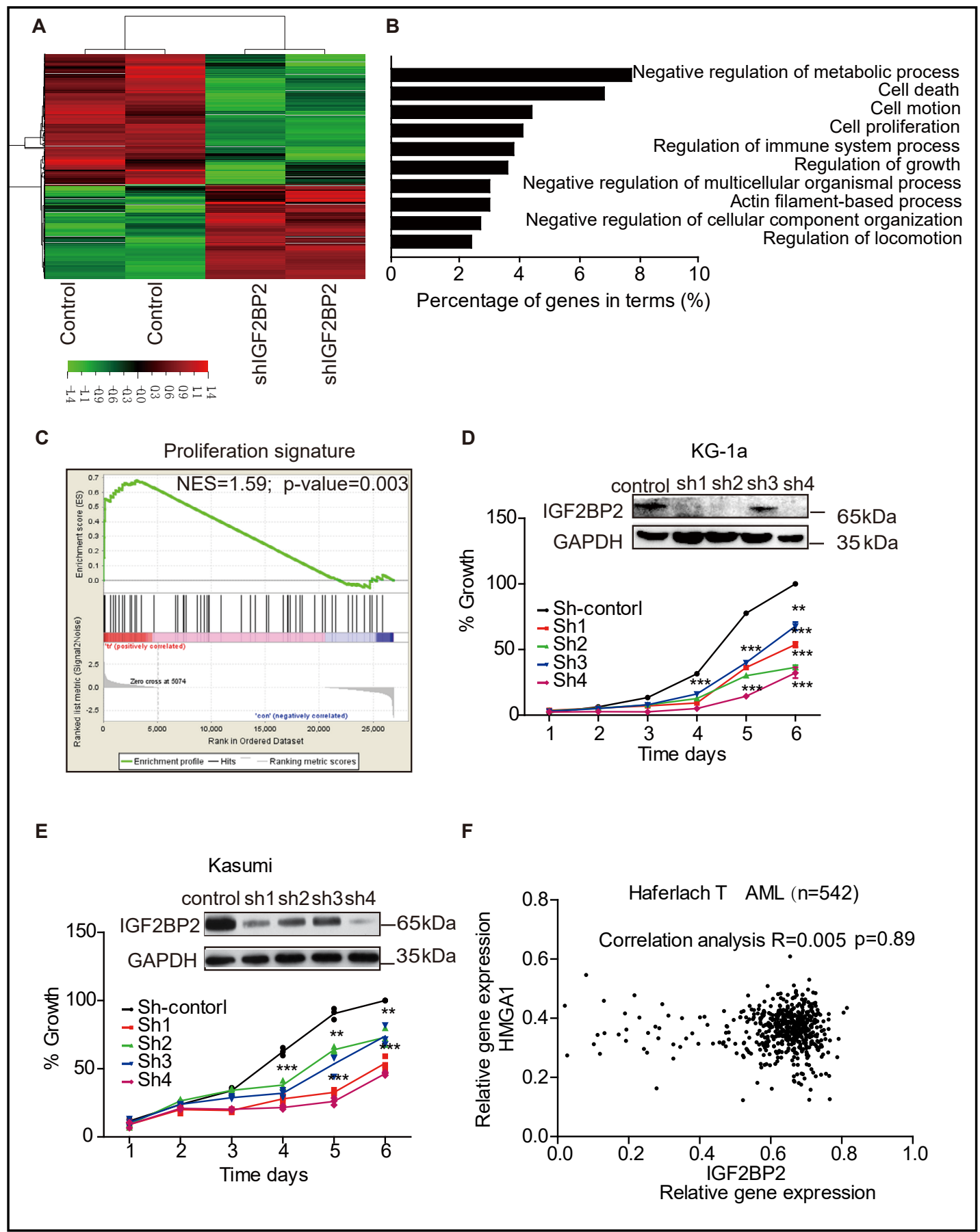

Fig. 5. Knockdown of IGF2BP2 reduces the growth and viability of AML cells. (A) Hierarchical clustering of gene expression profiles from a leukemia cell line with IGF2BP2 knockdown compared to control (GSE80946); (B) Gene Ontology terms analysis of the differential expression of genes between the IGF2BP2 knockdown and control groups; (C) Gene set enrichment analysis showing the enrichment of proliferation genes in the IGF2BP2 knockdown group. The normalized enrichment score (NES) and statistically significant p values are indicated; (D) IGF2BP2 knockdown inhibited the growth of the cell line KG-1a; (E) Knockdown of IGF2BP2 expression inhibited the growth of the cell line Kasumi; (F) co-expression analysis of the association of IGF2BP2 and HMGA1 in dataset(GSE13159). The results represent the mean \pm SEM; all experiments were performed in triplicate. ${ }^{* *} \mathrm{p}<0.01$, and ${ }^{* * *} \mathrm{p}<0.001$. 


\section{Cellular Physiology Cell Physiol Biochem 2018;51:1945-1956

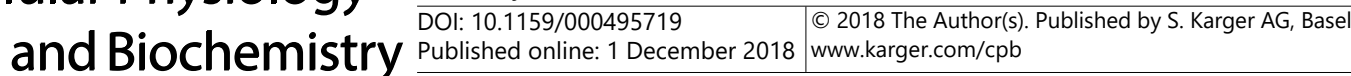 \\ He et al.: The Role of IGF2BP2 in Acute Myelocytic Leukemia}

had a poorer OS than the IGF2BP2 low expression group [HR=1.9(1.28-1.83), p<0.05] (Fig. $4 \mathrm{~A})$. The other two datasets also revealed that IGF2BP2 expression was correlated with poor OS [GSE8043: HR=1.63(1.05-2.52) p<0.05, GSE6891: HR=1.33(1.07-1.65) p<0.05]; however, in the GSE14468 dataset, the results were not statistically significant ( $p>0.05$ ) (Fig. 4B) [16]. Therefore, the correlation of IGF2BP2 expression with OS was inconclusive and controversial in different studies. To identify the prognostic value of IGF2BP2, we performed a systematic meta-analysis of eight studies consisting of 1731 patients. The pooled HR value for OS was 1.31 (95\% CI: 1.16-1.49; $\mathrm{Z}=4.27 ; \mathrm{p}=0.000$ ) (Fig. 4C) without heterogeneity $\left(\mathrm{I}^{2}=33.2 \%\right.$ $\mathrm{p}=0.163)$. This result indicated that the HR of overall death was 1.31-fold higher in AML patients with increased IGF2BP2 expression. In addition, both Egger's test ( $p=0.497)$ and Begg's test $(p=0.458)$ suggested that no publication bias existed (Fig. 4D). Taken together, these data indicate that IGF2BP2 overexpression is significantly associated with poor OS in AML patients.

Genetic knockdown of IGF2BP2 represses the growth of AML cell lines

To investigate the role of IGF2BP2 in AML, we searched the GEO database and found a gene expression profile dataset, GSE80946, in which IGF2BP2 was knocked down in a leukemia cell line (Fig. 5A). We downloaded the original data and performed a GO functional annotation analysis. The results showed that the changed genes were mainly enriched in cell growth and survival (Fig. 5B). The results of the Gene Set Enrichment Analysis (GSEA) also revealed a significant relation with proliferation (Fig. 5C). Thus, we transfected the cell lines KG-1a and Kasumi with an IGF2BP2 shRNA lentivirus and performed a proliferation assay. IGF2BP2 expression was verified by an immunoblotting assay. The results showed that IGF2BP2 shRNA caused obvious IGF2BP2 knockdown in the KG-1a and Kasumi cell lines. The proliferation assay showed that IGF2BP2 knockdown significantly inhibited the growth of the AML cell lines KG-1a and Kasumi (Fig. 5D-E). These results show that the knockdown of IGF2BP2 reduces cell growth in AML cell lines. It had been reported that IGF2BP2 can drive cancer cell proliferation by binding and stabilizing HMGA1 in various solid tumors [17]. We analyzed the relationship between IGF2BP2 and HMGA1 in AML using RNA-sequencing data. The result showed that the knockdown of IGF2BP2 did not significantly alter the expression of HMGA1 ( $p>0.05)$. In addition, we performed a co-expression analysis of a dataset that included $542 \mathrm{AML}$ patients (GSE13159) and found that IGF2BP2 was not correlated with the expression of HMGA1 (R=0.005, $\mathrm{p}=0.89$ ) (Fig. 5F). The mechanism of IGF2BP2 in maintaining AML proliferation still requires further investigation.

\section{Discussion}

IGF2BP2, a member of the conserved family of RNA-binding proteins, forms a complex termed the ribonucleoprotein complex and controls mRNA translation and transport [1820]. IGF2BP2 has also been suggested to be upregulated in many solid cancers, and it plays an important role in promoting the growth, migration and metastasis of cancer $[9,10,21$ 24]. However, the role of IGF2BP2 in AML has not been investigated systematically. In this study, we analyzed the differences in IGF2BP2 expression between AML patients and healthy controls by in silico analysis and demonstrated that IGF2BP2 is upregulated in AML patients; furthermore, IGF2BP2 expression was significantly correlated with worse prognosis in AML.

Previous studies have suggested that IGF2BP2 is upregulated in liposarcomas, endometrial adenocarcinomas and breast cancer [21-23]. A study had also reported that IGF2BP2 in acute lymphoblastic leukemia (ALL) is higher than in healthy donor bone marrow and peripheral blood, while IGF2BP1 and IGF2BP3 is downregulated in ALL[25]. More importantly, IGF2BP2 can serve as a prognostic indicator in several types of solid tumors, including esophageal adenocarcinoma, esophageal squamous carcinoma and glioblastoma [10,24]. Consistent with these data, we found that IGF2BP2 is overexpressed in primary AML patients and patient cohort datasets. In addition, IGF2BP2 overexpression 


\section{Cellular Physiology Cell Physiol Biochem 2018;51:1945-1956

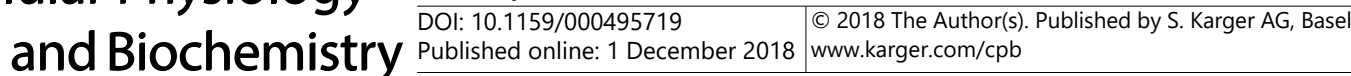 \\ He et al.: The Role of IGF2BP2 in Acute Myelocytic Leukemia}

is significantly associated with worse overall survival in AML patients and could act as a prognostic indicator for AML risk stratification. First, we analyzed the relationship between IGF2BP2 expression and worse clinical characteristics in AML patients (CEBPA, FLT3-ITD, and IDH1 mutation statuses as well as intermediate and poor cytogenetic risk). The FLT3ITD mutation is one of the most common genetic alterations in AML and is present in nearly $30 \%$ of AML patients $[26,27]$. AML patients with FLT3-ITD mutations have worse diseasefree and overall survivals [28]. IDH1 is also a frequent mutation in AML patients and confers an adverse prognosis in cytogenetically normal AML patients with the NPM1 mutation but without the FLT3-ITD mutation [29]. Biallelic CEBPA gene mutations represent a distinct molecular entity of cytogenetically normal AML patients, and patients with biallelic CEBPA gene mutations have better overall survival [30]. In this study, we demonstrated that IGF2BP2 was highly expressed in patients with FLT3-ITD and IDH1 mutations and was downregulated in patients with the CEBPA mutation. These results indicated that IGF2BP2 was associated with worse clinical characteristics in AML patients. Second, we performed a meta-analysis to investigate the prognostic significance of IGF2BP2 in AML for the first time. The pooled HR value for OS was 1.31 (95\% CI: $1.16-1.49 ; \mathrm{Z}=4.27$; $\mathrm{p}=0.00$ ), which indicated that IGF2BP2 overexpression was significantly associated with poor OS in AML patients. In addition, both Egger's test and Begg's test suggested that no publication bias existed in this meta-analysis.

Given the connection between high IGF2BP2 expression and AML prognosis, we then tested the role of IGF2BP2 in AML cell lines. Based on the GO and KEGG analyses of more than 60 esophageal adenocarcinoma patients, it has been reported that IGF2BP2 is significantly associated with proliferation and growth [10]. In addition, a recent study reported that the knockdown of IGF2BP2 expression in colorectal cancer cells suppressed cell growth [31]. Our results also showed that the knockdown of IGF2BP2 expression in AML cell lines obviously repressed cell growth. The mechanism of IGF2BP2 in maintaining AML proliferation still requires further investigation. More importantly, it has been suggested that IGF2BP2\% mice exhibit greater uncoupled oxygen consumption and a longer lifespan than wild-type mice. These results indicate that the lack of IGF2BP2 has no significant effect on normal cells. Therefore, IGF2BP2 may be a promising therapeutic target in AML.

\section{Conclusion}

In summary, our study results indicate that IGF2BP2 expression is upregulated in AML patients. Notably, IGF2BP2 overexpression is associated with worse clinical characteristics in AML patients (CEBPA, FLT3-ITD, and IDH1 mutation statuses as well as intermediate and poor cytogenetic risk) and is significantly associated with the poor overall survival of AML patients. In addition, the genetic knockdown of IGF2BP2 represses the growth of AML cell lines. Thus, IGF2BP2 may serve as a biomarker to predict the prognosis of AML and as a potential therapeutic target in AML patients.

\section{Acknowledgements}

This work is supported by the National Natural Science Foundation of China (NO.81502564 and NO. 81703498), Natural Science Foundation of Zhejiang province (NO. LY16H160018), and scholarship from China Scholarship Council (CSC) under the Grant CSC (NO. 201706325003). The study followed the Declaration of Helsinki principles and was approved by the Ethics Committee of the Second Affiliated Hospital of Zhejiang University.

\section{Disclosure Statement}

The authors declared that they have no conflicts of interest to this work.

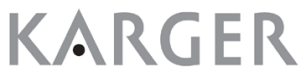




\section{Cellular Physiology Cell Physiol Biochem 2018;51:1945-1956

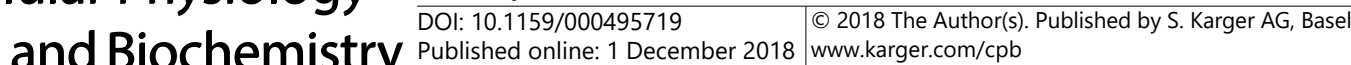

\section{References}

1 Byrd JC, Mrozek K, Dodge RK, Carroll AJ, Edwards CG, Arthur DC, Pettenati MJ, Patil SR, Rao KW, Watson MS, Koduru PR, Moore JO, Stone RM, Mayer RJ, Feldman EJ, Davey FR, Schiffer CA, Larson RA, Bloomfield CD, Cancer, Leukemia Group B: Pretreatment cytogenetic abnormalities are predictive of induction success, cumulative incidence of relapse, and overall survival in adult patients with de novo acute myeloid leukemia: results from Cancer and Leukemia Group B (CALGB 8461). Blood 2002;100:4325-4336.

-2 Valk PJ, Verhaak RG, Beijen MA, Erpelinck CA, Barjesteh van Waalwijk van Doorn-Khosrovani S, Boer JM, Beverloo HB, Moorhouse MJ, van der Spek PJ, Lowenberg B, Delwel R: Prognostically useful geneexpression profiles in acute myeloid leukemia. N Engl J Med 2004;350:1617-1628.

$\rightarrow 3$ Siegel RL, Miller KD, Jemal A: Cancer statistics, 2016. CA Cancer J Clin 2016;66:7-30.

$>4$ Schlenk RF, Dohner K, Krauter J, Frohling S, Corbacioglu A, Bullinger L, Habdank M, Spath D, Morgan M, Benner A, Schlegelberger B, Heil G, Ganser A, Dohner H, German-Austrian Acute Myeloid Leukemia Study G: Mutations and treatment outcome in cytogenetically normal acute myeloid leukemia. N Engl J Med 2008;358:1909-1918.

5 Patel JP, Gonen M, Figueroa ME, Fernandez H, Sun Z, Racevskis J, Van Vlierberghe P, Dolgalev I, Thomas S, Aminova O, Huberman K, Cheng J, Viale A, Socci ND, Heguy A, Cherry A, Vance G, Higgins RR, Ketterling RP, Gallagher RE et al.: Prognostic relevance of integrated genetic profiling in acute myeloid leukemia. N Engl J Med 2012;366:1079-1089.

6 Fujii Y, Kishi Y, Gotoh Y: IMP2 regulates differentiation potentials of mouse neocortical neural precursor cells. Genes Cells 2013;18:79-89.

7 Li Z, Gilbert JA, Zhang Y, Zhang M, Qiu Q, Ramanujan K, Shavlakadze T, Eash JK, Scaramozza A, Goddeeris MM, Kirsch DG, Campbell KP, Brack AS, Glass DJ: An HMGA2-IGF2BP2 axis regulates myoblast proliferation and myogenesis. Dev Cell 2012;23:1176-1188.

8 Boudoukha S, Cuvellier S, Polesskaya A: Role of the RNA-binding protein IMP-2 in muscle cell motility. Mol Cell Biol 2010;30:5710-5725.

-9 Barghash A, Helms V, Kessler SM: Overexpression of IGF2 mRNA-Binding Protein 2 (IMP2/p62) as a Feature of Basal-like Breast Cancer Correlates with Short Survival. Scand J Immunol 2015;82:142-143.

$>10$ Barghash A, Golob-Schwarzl N, Helms V, Haybaeck J, Kessler SM: Elevated expression of the IGF2 mRNA binding protein 2 (IGF2BP2/IMP2) is linked to short survival and metastasis in esophageal adenocarcinoma. Oncotarget 2016;10.18632/oncotarget.10439

11 Mu Q, Wang L, Yu F, Gao H, Lei T, Li P, Liu P, Zheng X, Hu X, Chen Y, Jiang Z, Sayari AJ, Shen J, Huang H: Imp2 regulates GBM progression by activating IGF2/PI3K/Akt pathway. Cancer Biol Ther 2015;16:623-633.

12 Kessler SM, Laggai S, Barghash A, Schultheiss CS, Lederer E, Artl M, Helms V, Haybaeck J, Kiemer AK: IMP2/ p62 induces genomic instability and an aggressive hepatocellular carcinoma phenotype. Cell Death Dis 2015;6:e1894.

13 de Jonge HJ, Valk PJ, Veeger NJ, ter Elst A, den Boer ML, Cloos J, de Haas V, van den Heuvel-Eibrink MM, Kaspers GJ, Zwaan CM, Kamps WA, Lowenberg B, de Bont ES: High VEGFC expression is associated with unique gene expression profiles and predicts adverse prognosis in pediatric and adult acute myeloid leukemia. Blood 2010;116:1747-1754.

-14 Verhaak RG, Wouters BJ, Erpelinck CA, Abbas S, Beverloo HB, Lugthart S, Lowenberg B, Delwel R, Valk PJ: Prediction of molecular subtypes in acute myeloid leukemia based on gene expression profiling. Haematologica 2009;94:131-134.

-15 Tomasson MH, Xiang Z, Walgren R, Zhao Y, Kasai Y, Miner T, Ries RE, Lubman O, Fremont DH, McLellan MD, Payton JE, Westervelt P, DiPersio JF, Link DC, Walter MJ, Graubert TA, Watson M, Baty J, Heath S, Shannon WD et al.: Somatic mutations and germline sequence variants in the expressed tyrosine kinase genes of patients with de novo acute myeloid leukemia. Blood 2008;111:4797-4808.

16 Wouters BJ, Lowenberg B, Erpelinck-Verschueren CA, van Putten WL, Valk PJ, Delwel R: Double CEBPA mutations, but not single CEBPA mutations, define a subgroup of acute myeloid leukemia with a distinctive gene expression profile that is uniquely associated with a favorable outcome. Blood 2009;113:3088-3091.

-17 Dai N, Ji F, Wright J, Minichiello L, Sadreyev R, Avruch J: IGF2 mRNA binding protein-2 is a tumor promoter that drives cancer proliferation through its client mRNAs IGF2 and HMGA1. Elife 2017;6:e27155. 


\section{Cellular Physiology Cell Physiol Biochem 2018;51:1945-1956 and Biochemistry DOl: 10.1159/000495719 2018 合 2018 The Author(s). Published by S. Karger AG, Basel

He et al.: The Role of IGF2BP2 in Acute Myelocytic Leukemia

18 Nielsen J, Christiansen J, Lykke-Andersen J, Johnsen AH, Wewer UM, Nielsen FC: A family of insulinlike growth factor II mRNA-binding proteins represses translation in late development. Mol Cell Biol 1999;19:1262-1270.

19 Runge S, Nielsen FC, Nielsen J, Lykke-Andersen J, Wewer UM, Christiansen J: H19 RNA binds four molecules of insulin-like growth factor II mRNA-binding protein. J Biol Chem 2000;275:29562-29569.

-20 Bell JL, Wachter K, Muhleck B, Pazaitis N, Kohn M, Lederer M, Huttelmaier S: Insulin-like growth factor 2 mRNA-binding proteins (IGF2BPs): post-transcriptional drivers of cancer progression? Cell Mol Life Sci 2013;70:2657-2675.

21 Zhang L, Liu Y, Hao S, Woda BA, Lu D: IMP2 expression distinguishes endometrioid from serous endometrial adenocarcinomas. Am J Surg Pathol 2011;35:868-872.

-22 Li Y, Francia G, Zhang JY: p62/IMP2 stimulates cell migration and reduces cell adhesion in breast cancer. Oncotarget 2015;6:32656-32668.

23 Cleynen I, Brants JR, Peeters K, Deckers R, Debiec-Rychter M, Sciot R, Van de Ven WJ, Petit MM: HMGA2 regulates transcription of the Imp2 gene via an intronic regulatory element in cooperation with nuclear factor-kappaB. Mol Cancer Res 2007;5:363-372.

24 Janiszewska M, Suva ML, Riggi N, Houtkooper RH, Auwerx J, Clement-Schatlo V, Radovanovic I, Rheinbay E, Provero P, Stamenkovic I: Imp2 controls oxidative phosphorylation and is crucial for preserving glioblastoma cancer stem cells. Genes Dev 2012;26:1926-1944.

-25 Stoskus M, Gineikiene E, Valceckiene V, Valatkaite B, Pileckyte R, Griskevicius L: Identification of characteristic IGF2BP expression patterns in distinct B-ALL entities. Blood Cells Mol Dis 2011;46:321-326.

-26 Frohling S, Schlenk RF, Breitruck J, Benner A, Kreitmeier S, Tobis K, Dohner H, Dohner K, leukemia AMLSGUAm: Prognostic significance of activating FLT3 mutations in younger adults (16 to 60 years) with acute myeloid leukemia and normal cytogenetics: a study of the AML Study Group Ulm. Blood 2002;100:4372-4380.

-27 Schnittger S, Schoch C, Dugas M, Kern W, Staib P, Wuchter C, Loffler H, Sauerland CM, Serve H, Buchner T, Haferlach T, Hiddemann W: Analysis of FLT3 length mutations in 1003 patients with acute myeloid leukemia: correlation to cytogenetics, FAB subtype, and prognosis in the AMLCG study and usefulness as a marker for the detection of minimal residual disease. Blood 2002;100:59-66.

28 Sheikhha MH, Awan A, Tobal K, Liu Yin JA: Prognostic significance of FLT3 ITD and D835 mutations in AML patients. Hematol J 2003;4:41-46.

29 Paschka P, Schlenk RF, Gaidzik VI, Habdank M, Kronke J, Bullinger L, Spath D, Kayser S, Zucknick M, Gotze K, Horst HA, Germing U, Dohner H, Dohner K: IDH1 and IDH2 mutations are frequent genetic alterations in acute myeloid leukemia and confer adverse prognosis in cytogenetically normal acute myeloid leukemia with NPM1 mutation without FLT3 internal tandem duplication. J Clin Oncol 2010;28:3636-3643.

-30 Dufour A, Schneider F, Metzeler KH, Hoster E, Schneider S, Zellmeier E, Benthaus T, Sauerland MC, Berdel WE, Buchner T, Wormann B, Braess J, Hiddemann W, Bohlander SK, Spiekermann K: Acute myeloid leukemia with biallelic CEBPA gene mutations and normal karyotype represents a distinct genetic entity associated with a favorable clinical outcome. J Clin Oncol 2010;28:570-577.

-31 Ye S, Song W, Xu X, Zhao X, Yang L: IGF2BP2 promotes colorectal cancer cell proliferation and survival through interfering with RAF-1 degradation by miR-195. FEBS Lett 2016;590:1641-1650.

32 Gentles AJ, Newman AM, Liu CL, Bratman SV, Feng W, Kim D, Nair VS, Xu Y, Khuong A, Hoang CD, Diehn M, West RB, Plevritis SK, Alizadeh AA: The prognostic landscape of genes and infiltrating immune cells across human cancers. Nat Med 2015;21:938-945.

-33 Higgins JP, Thompson SG, Deeks JJ, Altman DG: Measuring inconsistency in meta-analyses. BMJ 2003;327:557-560.

34 Mantel N, Haenszel W: Statistical aspects of the analysis of data from retrospective studies of disease. J Natl Cancer Inst 1959;22:719-748. 